\title{
Transoral robotic biopsy of the tongue base: A novel paradigm in the evaluation of unknown primary tumors of the head and neck
}

\author{
Waleed M. Abuzeid, MBBS, Carol R. Bradford, MD, Vasu Divi, MD \\ Department of Otolaryngology: Head and Neck Surgery, University of Michigan, Ann Arbor, Michigan 48109. \\ Accepted 7 September 2011 \\ Published online 16 December 2011 in Wiley Online Library (wileyonlinelibrary.com). DOI 10.1002/hed.21968
}

ABSTRACT: Background. Squamous cell carcinoma of the head and neck can present as a cervical metastasis from an unknown primary site. The standard diagnostic workup includes panendoscopy and directed biopsies but this will fail to identify a portion of unknown primary tumors.

Methods. Herein, we present a case report of a male patient with an unknown primary tumor in which the da Vinci surgical robot was used to evaluate the tongue base.

Results. Clinical evaluation, imaging, and panendoscopy with directed biopsies failed to detect the primary tumor site. Robot-assisted biopsy of a broad area of the tongue base, incorporating submucosal tissue, identified the primary tumor with minimal postoperative morbidity.

Conclusion. Failure to localize an unknown primary tumor often results in widespread irradiation of the upper aerodigestive tract, inducing significant morbidity. Robot-assisted biopsies of the tongue base may identify unknown primaries that would otherwise have been missed through standard directed biopsy techniques. (C) 2011 Wiley Periodicals, Inc. Head Neck 35: E126-E130, 2013

KEY WORDS: carcinoma, diagnosis, robotic, unknown, biopsy
Approximately $2 \%$ to $5 \%$ of squamous cell carcinomas of the head and neck present as a cervical metastasis from an unknown primary tumor site. ${ }^{1}$ Localization of the unknown primary tumor remains a challenge, with fewer than $60 \%$ of primary tumors eventually being discovered. $^{2}$ The primary site, when identified, is usually in the upper aerodigestive tract, specifically the palatine tonsils, base of tongue, and nasopharynx. Small tumors originating in these areas can be missed by clinical and radiographic assessment due to the inherent anatomic complexity of these regions and limitations of currently used diagnostic techniques. ${ }^{3}$

To be designated as a true unknown primary tumor, patients should undergo a comprehensive evaluation by an experienced head and neck surgeon. The evaluation begins with a thorough head and neck physical examination followed by fiber-optic endoscopy of the nasopharynx, oropharynx, hypopharynx, and larynx. ${ }^{1,2}$ Between $52 \%$ and $55 \%$ of unknown primaries are identified on the basis of history and physical examination alone. ${ }^{2}$

Contrast-enhanced CT and/or MRI with gadolinium are an integral component of the workup for the unknown primary. Use of either modality will result in detection of occult primary sites in $17 \%$ to $31 \%$ of cases. ${ }^{2,3}$ Positron emission tomography (PET), either alone or fused with

${ }^{*}$ Corresponding author: V. Divi, Department of Otolaryngology: Head and Neck Surgery, University of Michigan, Ann Arbor, M ichigan 48109.

E-mail: vasudivi@med.umich.edu
CT imaging (PET-CT), has been advocated as an adjunctive modality where CT and/or MRI fail to yield an apparent primary site. A recent retrospective study demonstrated that PET-CT has a detection rate of $36.8 \%$, which is higher than the PET detection rate of $25 \%$ averaged from 16 studies. ${ }^{4}$ Although PET-CT demonstrates improved specificity over PET (95\% vs $75 \%$ ), it remains susceptible to false positives due to higher metabolism in the lymphatic tissue of Waldeyer's ring, including the palatine and lingual tonsils. ${ }^{4}$

Panendoscopy is a critical component of the diagnostic workup and remains the gold standard for the identification of unknown primary tumors. Panendoscopy can detect previously occult primary tumors in $16 \%$ to $26 \%$ of cases. ${ }^{2}$ However, mucosal abnormalities suggestive of malignancy are often not seen on panendoscopy leading to the practice of "directed" biopsies in the nasopharynx, tongue base, tonsils, and pyriform sinuses, even in the absence of a visual abnormality. In 1 series, 9\% of occult tumors were diagnosed on the basis of these random or directed biopsies. ${ }^{2,5}$ The tongue base is the most common site harboring unknown primary tumors. ${ }^{2}$ In contrast to the tonsil, a complete biopsy of the entire base of tongue is technically difficult with traditional instruments. As a result, palpation of the tongue base and visualization of subtle mucosal irregularities or areas of friability direct biopsies toward focal areas of the tongue base.

We have recently used the da Vinci surgical robot (Intuitive Surgical, Sunnyvale, CA) to facilitate biopsies of the tongue base in cases of an unknown primary tumor. The enhanced access to the base of tongue allows us to 


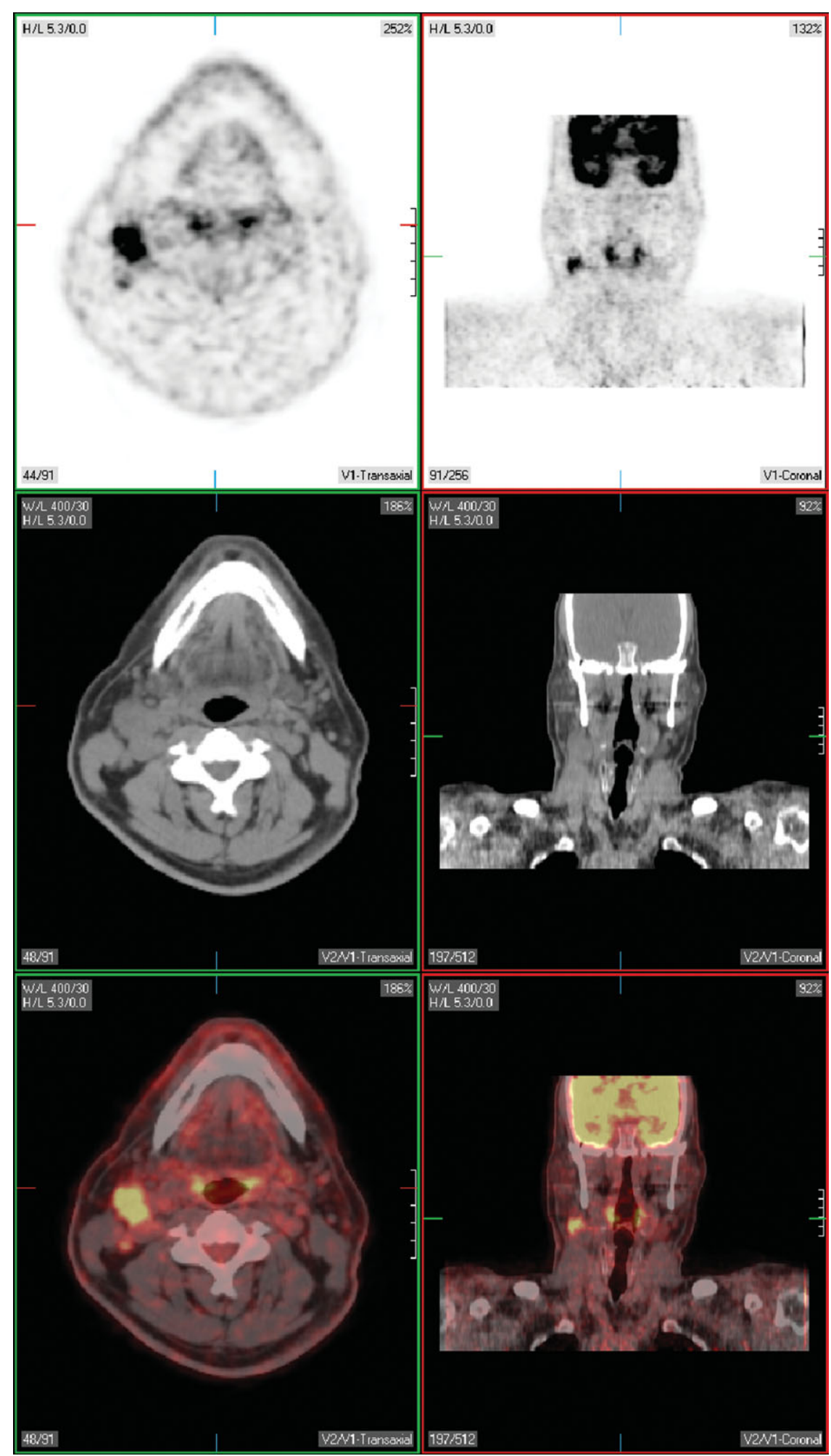

FIGURE 1. Discrete and fused positron emission tomography-CT (PET-CT) images demonstrate an enlarged, necrotic, right level II/III lymph node (4 $\times 2.8 \times 2.5 \mathrm{~cm}$ ) with intensely increased fluorodeoxyglucose (FDG) uptake. Immediately posterior to this mass is a smaller, level Ilb lymph node with mild FDG avidity. The distribution of FDG in the remainder of the head and neck is within physiologic limits. In particular, no abnormal FDG uptake is visualized in the tongue base. 


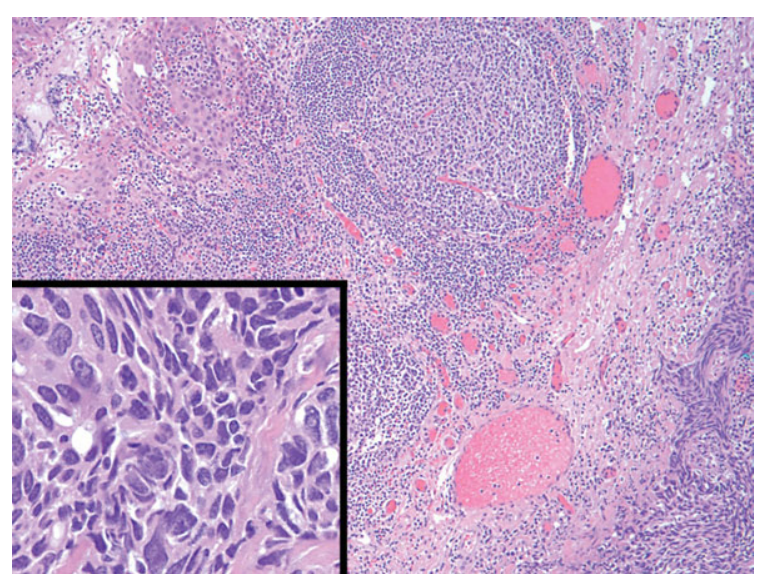

FIGURE 2. Hematoxylin-eosin staining of tongue base biopsy (original magnification $\times 100$ ) demonstrating squamous cell carcinoma in the bottom right with overlying normal tonsillar tissue in the upper left of the main image. The inset represents the identified squamous cell carcinoma (original magnification $\times 400$ ).

obtain a biopsy covering a greater mucosal surface area without significant morbidity. This case report illustrates this novel paradigm.

\section{CASE REPORT}

A 57-year-old man presented to our clinic for evaluation of an asymptomatic right neck mass. The patient had a 30 pack-year smoking history but had quit 16 years earlier. He had a tonsillectomy during childhood. The right neck mass had been noticed 6 weeks before presentation and was treated with several courses of antibiotics by outside physicians. A CT scan requested by the referring otolaryngologist demonstrated a $4 \times 2.8 \times 2.5 \mathrm{~cm}$ mass in level II/III of the right neck. Fine-needle aspiration of this mass demonstrated metastatic squamous cell carcinoma. A PET scan ordered by the outside surgeon showed a necrotic, intensely fluorodeoxyglucose (FDG)-avid lymph node, corresponding to the lesion seen on CT, and several smaller FDG-avid level IIb nodes but no primary tumor (Figure 1). Our physical examination, including flexible laryngoscopy, failed to yield a primary tumor site. Neck palpation did demonstrate an obvious soft, mobile level II/III lymph node. The patient was classified as having a TxN2bM0 squamous cell carcinoma of the right neck.

The patient was taken to the operating room for panendoscopy and directed biopsies after providing written informed consent. Palpation of the oropharynx revealed a firm nodule in the right tongue base. On direct laryngoscopy, a pale, raised area measuring approximately $5 \mathrm{~mm}$ in diameter was seen in the inferior right tongue base, corresponding to the previously palpated area. To better define this site, microdirect laryngoscopy was performed using suspension laryngoscopy and a $0^{\circ}$ rigid endoscope attached to a high definition video system. Precise biopsies of the seemingly abnormal area and additional biopsies in the surrounding mucosa and glossotonsillar sulcus were taken and sent for frozen section biopsies. All the biopsies were negative for malignancy. An unremarkable esophagoscopy was then performed. Nasopharyngoscopy was then performed using a rigid endoscope which revealed no lesions. Frozen-section biopsies of the bilateral nasopharynx were negative for malignancy. Given the failure to elucidate the primary tumor site on panendoscopy, we proceeded with transoral robotic biopsy of the right lingual tonsil. A Crowe-Davis mouth gag was inserted and the patient was placed in suspension to optimize tongue base visualization. The da Vinci robot was positioned and the robotic arms placed transorally, as previously described. ${ }^{6}$ An incision was made perpendicular to the midline to define the anterior extent of the biopsy, just posterior to the circumvallate papillae. This incision was continued to the midline where a longitudinal incision was made to define the medial border. This created a tissue edge which was then retracted with the Maryland dissector. A lateral cut was made just medial to the lateral pharyngeal wall. Electrocautery was used to remove the superficial portion of the lingual tonsil extending from the circumvallate papillae to just proximal to the vallecula. Once the mucosa was circumferentially resected, it was oriented and passed off the field for permanent pathology. The patient's neck disease was then addressed with an uncomplicated selective neck dissection incorporating levels I to IV. The patient was extubated at the end of the surgery and, after recovery in the postoperative care unit, was transferred to the general care floor.

Postoperatively, the patient did not demonstrate any respiratory difficulties. He was advanced to a full liquid diet on postoperative day 0 with no aspiration noted. He was discharged home on postoperative day 2 , returning to the clinic on postoperative day 8 for follow-up. At that time, he reported mild odynophagia and he was advanced to a regular diet. He was diagnosed with oral candidiasis and started on nystatin swish and swallow. Pathologic analysis of the operative specimens confirmed that the traditional directed biopsies of the tongue base and nasopharynx were negative for malignancy. The right lingual tonsil resection, however, demonstrated a focus of human papillomavirus-positive invasive squamous cell carcinoma in the deep tissue at the inferior/medial margin with no abnormality noted in the overlying squamous mucosa (Figure 2). The right neck dissection yielded 34 lymph nodes, of which a single 3.2-cm level III lymph node, corresponding to the patient's neck mass, was positive for carcinoma with no evidence of extracapsular extension. These results were discussed at our multidisciplinary head and neck tumor board, and the tumor was reclassified as pT1N2aM0. Because the primary lesion was close to the midline base of tongue, it was felt that the patient would need treatment to the bilateral necks. While definitive resection of the central tongue base and contralateral neck dissection remained an option, the tumor board recommended radiation to the primary site and to the bilateral necks as optimal treatment.

\section{DISCUSSION}

The treatment of the unknown primary tumor varies between different institutions. The most commonly used 




strategies include upfront concurrent chemoradiation versus neck dissection followed by adjuvant radiation or chemoradiation based on the pathology results. Identification of an unknown primary tumor can significantly influence patient treatment and side effects.

In patients with $\mathrm{N} 1$ and some $\mathrm{N} 2 \mathrm{a}$ neck disease in whom the primary tumor has been identified in the oropharynx, treatment may allow for surgical extirpation of the primary and neck dissection without adjuvant chemotherapy or radiation. In cases in which adverse pathologic features are present, patients require adjuvant treatment. However, the radiation doses are reduced and can be focused on the primary tumor site, as opposed to covering all potential primary sites. Those with more advanced neck disease (N2/N3) and no evidence of extracapsular spread can also be treated with unilateral comprehensive neck dissection followed by bilateral neck irradiation and targeted mucosal irradiation of the oropharynx. In these patients, identifying the primary tumor and performing a neck dissection again offers focused radiation and the potential to avoid chemotherapy as part of their treatment.
Currently, up-front concurrent chemoradiation is an increasingly used option even in these patients. ${ }^{7,8}$

The identification of an unknown primary tumor site can potentially spare the patient the significant morbidity associated with concurrent chemoradiation. In a recent study investigating the treatment of unknown primaries of the head and neck, the incidence of grade $3+$ acute toxicity (most commonly severe, confluent mucositis) was $59 \%$ among patients treated with chemoradiation versus $25 \%$ among those treated with radiation alone $(p<$ $.001){ }^{8}$ The risk of grade $3+$ acute esophagitis was also significantly increased in the chemoradiation group relative to the radiation therapy alone group (47\% vs $21 \%$; $p<$ $.001)$. Late effects were also significantly higher in the chemoradiation group versus the radiation alone group. These late effects included dysphagia secondary to esophageal toxicity $(41 \%$ vs $11 \% ; p<.001)$, esophageal structure (34\% vs $7 \% ; p<.001)$, and gastrostomy tube dependence at 1 year $(16 \%$ vs $0 \% ; p<.001)$. Nearly $20 \%$ of the patients treated with concurrent chemoradiation required hospitalization for acute renal failure, intractable nausea/ 
vomiting, and neutropenic fever, among other reasons, compared to only $4 \%$ treated with radiation alone. ${ }^{8}$

Even in those institutions that elect to forego chemotherapy and instead use comprehensive radiation of mucosal sites commonly harboring occult primary tumors and the bilateral necks, the resultant toxicity can be impressive. These toxicities include a high incidence of xerostomia, mucositis, skin damage, dysphagia, and stricture. ${ }^{2}$ More advanced intensity-modulated radiotherapy (IMRT) regimens have improved upon the toxicity of conventional radiotherapy but still induce significant morbidity. In 1 study from Memorial Sloan-Kettering Cancer Center, IMRT, when compared with conventional radiotherapy, significantly reduced the risk of grade 1 to 2 xerostomia ( $57 \%$ vs $43 \%$, respectively) and in gastrostomy rates ( $72 \%$ vs $43 \%$, respectively). ${ }^{9}$ Nonetheless, these risks remain markedly higher than those associated with IMRT directed to an identified primary site and unilateral or bilateral necks. To illustrate, had the primary site not been identified in the index case discussed above, the presence of N2 disease would have resulted in comprehensive radiation of the mucosal surfaces of the upper aerodigestive tract, the nasopharynx or retropharyngeal nodes, and the bilateral necks, with concurrent chemotherapy. Thus, techniques that may facilitate the detection of the primary tumor are particularly pertinent.

There is an increased incidence of human papillomavirus-associated oropharyngeal carcinoma located in the tonsillar or base of tongue tissue, which tend to present with larger neck disease and smaller primary tumors. If this does lead to an increased incidence of unknown primary tumors, better evaluation of the base of tongue would aid in localizing this disease. Transoral robotic surgery allows for a superficial "pancake', biopsy incorporating the entirety of the suspected tongue base. This permits pathologic analysis of a larger volume of tongue base tissue, potentially enhancing the probability of detecting the occult primary tumor. This may be of particular value in the scenario described in our index case where standard biopsies via microdirect laryngoscopy were negative or where a discrete abnormality is not noted on routine endoscopy. Furthermore, as in the index case, the primary site may be submucosal with histologically "normal" overlying squamous mucosa (Figure 2). The controlled depth of the excisional biopsy limits functional morbidity and abbreviates hospital stay, but allows for pathologic evaluation of this submucosal disease.

We propose a novel algorithm for the workup of the unknown primary tumor that incorporates use of the da Vinci surgical platform (Figure 3). The initial preoperative workup and imaging studies are essentially unchanged from those described above. The patient is then brought to the operating room for panendoscopy and biopsies. The highest yield site for biopsies is the tongue base, which is the most likely site harboring an unknown primary tumor. ${ }^{3}$ Direct or microdirect laryngoscopy with biopsies should be performed. If the intraoperative frozen section is negative for malignancy, the da Vinci surgical robot, in the context of our proposed algorithm, is used for transoral tongue base biopsy, with excision of a wide "pancake" specimen. This specimen is sent for permanent pathology. Additional biopsies are taken, as indicated, from the nasopharynx and a tonsillectomy is performed. A unilateral selective neck dissection may be considered to provide lymph node tissue for evaluation of metastasis and determination of extracapsular spread. This will allow for treatment stratification per National Comprehensive Cancer Network guidelines should the primary tumor not be detected on the robotic tongue base biopsy.

The detection of the primary site in cases of unknown primaries of the head and neck is a management priority. Use of the da Vinci robot to obtain a "pancake" biopsy of the most common site of these occult primaries, the tongue base, may facilitate detection of primary tumors relative to standardly used panendoscopy techniques. The key benefit derived from establishing the diagnosis is the avoidance of wide radiation fields used for the treatment of unknown primaries and the potential avoidance of concurrent chemotherapy. Clinical trials will need to be designed to investigate the efficacy of transoral robotic surgery tongue base biopsy in identifying the primary site of disease in occult head and neck malignancies. Until these definitive studies are performed, this technique should be viewed as a management option, but should not be considered standard of care.

\section{REFERENCES}

1. Waltonen JD, Ozer E, Hall NC, Schuller DE, Agrawal A. Metastatic carcinoma of the neck of unknown primary origin: evolution and efficacy of the modern workup. Arch Otolaryngol Head Neck Surg 2009;135:1024-1029.

2. Galer CE, Kies MS. Evaluation and management of the unknown primary carcinoma of the head and neck. J Natl Compr Canc Netw 2008;6: $1068-1075$

3. Cianchetti M, Mancuso AA, Amdur RJ, et al. Diagnostic evaluation of squamous cell carcinoma metastatic to cervical lymph nodes from an unknown head and neck primary site. Laryngoscope 2009;119:2348-2354.

4. Keller F, Psychogios G, Linke R, et al. Carcinoma of unknown primary in the head and neck: comparison between positron emission tomography (PET) and PET/CT. Head Neck 2010 [Epub ahead of print].

5. Lee DJ, Rostock RA, Harris A, Kashima H, Johns M. Clinical evaluation of patients with metastatic squamous carcinoma of the neck with occult primary tumor. South Med J 1986;79:979-983.

6. O'Malley BW Jr, Weinstein GS, Snyder W, Hockstein NG. Transoral robotic surgery (TORS) for base of tongue neoplasms. Laryngoscope 2006;116: $1465-1472$.

7. Pfister DG, Ang K-K, Brizel D, et al. NCCN Practice Guidelines for Head and Neck Cancer. 2011

8. Chen AM, Farwell DG, Lau DH, Li BQ, Luu Q, Donald PJ. Radiation therapy in the management of head-and-neck cancer of unknown primary origin: how does the addition of concurrent chemotherapy affect the therapeutic ratio? Int J Radiat Oncol Biol Phys 2011;81:346-352.

9. Klem ML, Mechalakos JG, Wolden SL, et al. Intensity-modulated radiotherapy for head and neck cancer of unknown primary: toxicity and preliminary efficacy. Int J Radiat Oncol Biol Phys 2008;70: 1100-1107. 